\title{
Effects of puerarin on the retina and STAT3 expression in diabetic rats
}

\author{
YONGHAO CAI ${ }^{*}$, XIAOBI ZHANG ${ }^{*}$, XUEGU XU and YINFEI YU \\ The Eye Hospital of Wenzhou Medical University, Wenzhou, Zhejiang 325000, P.R. China
}

Received May 5, 2017; Accepted September 11, 2017

DOI:10.3892/etm.2017.5203

\begin{abstract}
The effects of puerarin on electroretinogram, oxidative stress and STAT3 expression were determined, in diabetic rat retina and serum. Forty Sprague-Dawley rats were randomly divided into the normal control (NC), the diabetic model (DM), the low dose $(250 \mathrm{mg} / \mathrm{kg})$ puerarin (LP) or the high dose $(500 \mathrm{mg} / \mathrm{kg})$ puerarin group (HP). A diabetic rat model was induced by streptozotocin and animals were continuously treated for 4 weeks; fasting blood glucose was measured at 2 and 4 weeks after modeling. An electroretinogram and serum and tissue levels of glucose, insulin, superoxide dismutase (SOD), malondialdehyde (MDA) and total antioxidant capacity (T-AOC) were measured; real-time PCR and ELISA were used to determine STAT3 mRNA and protein expression, respectively, from the retina. The blood glucose and insulin levels in the puerarin groups were significantly lower and higher, respectively than that in the DM group. The amplitude of b-wave of electroretinogram in the DM and the LP groups was significantly lower than that in the NC group; in the LP and HP groups it was significantly higher than the DM group. The serum and retinal tissue activity of SOD and MDA was significantly lower and higher, respectively, in the DM group compared to the NC group; both the LP and HP groups had significantly higher SOD and lower MDA than the DM group. The levels of STAT3 mRNA and protein levels in the DM, LP and HP groups were significantly higher than the NC group; and levels of STAT3 mRNA and protein expression were significantly lower in the LP and HP groups than the DM group. In summary, puerarin can reduce the oxidative stress damage of the retina, and its mechanism is related to the inhibition of STAT3 expression.
\end{abstract}

Correspondence to: Dr Yinfei Yu, The Eye Hospital of Wenzhou Medical University, 270 Xueyuan Road West, Wenzhou, Zhejiang 325000, P.R. China

E-mail: yinfei_yu@hotmail.com

*Contributed equally

Key words: puerarin, diabetic retinopathy, rat, oxidative stress, STAT3

\section{Introduction}

Diabetic retinopathy (DR), is a major complication of retinal vascular disease in patients with diabetes mellitus, which can result in blindness (1). Epidemiological studies have reported that approximately 250 million people worldwide suffer from diabetes; in rural areas of China, approximately $43 \%$ of patients have varying degrees of DR, of which $6.3 \%$ suffer from severe visual impairment (2). In the US population, approximately $86 \%$ of patients with type 1 diabetes and $40 \%$ of patients with type 2 diabetes have varying degrees of DR $(3,4)$. A high serum glucose state can cause damage to the retina, retinal capillary cell inflammation, oxidative stress and other reactions leading to abnormal capillary function, ischemia and eventual angiogenesis $(1,5)$.

Signal transducer and activator of transcription 3 (STAT3), is one of the members of a family of signal transducers and activators of transcription, that function by regulating cell growth, differentiation and angiogenesis and participates in the pathogenesis of DR (6). Given the sizeable number of patients with diabetes and the prevalence of DR, other than actively controlling serum glucose levels, there is no effective treatment that can reverse DR progression (7). Pueraria is a leguminous perennial plant, which contains both starch and flavonoids. The highest amount of the active flavonoid ingredient is puerarin; which has lipid-lowering, anti-oxidation, oxygen free radical scavenging and other pharmacological effects (8). Puerarin, has been shown to be effective in the treatment of cerebral ischemia (9), angina, myocardial infarction (10) and other ischemic diseases. In recent years, many studies have reported $(11,12)$ that puerarin has a positive effect in the treatment of diabetes and its complications. Therefore, in this study, a diabetic mouse model was induced by streptozotocin (STZ), and the protective effect of puerarin on DR was observed by measuring changes of blood glucose, oxidative stress and STAT3 expression; its mechanism of action was also studied for future potential clinical application of puerarin.

\section{Materials and methods}

Forty specific pathogen free (SPF)-grade Sprague-Dawley rats of 8-10 weeks of age, half male and half female, weight 200-220 g, were purchased from the Shanghai Lake Experimental Animal Center [license number: SCXK (Shanghai) 2012-0002]. Each animal with a normal fundus 
Table I. Primer sequences.

\begin{tabular}{lcl}
\hline Gene & Length $(\mathrm{bp})$ & \multicolumn{1}{c}{ Primer sequence } \\
\hline STAT3 & 112 & Forward: 5'-CACCTTGGATTGAGAGTCAAGAC-3' \\
& 95 & $\begin{array}{l}\text { Reverse: 5'-AGGAATCGGCTATATTGCTGGT-3' } \\
\text { GAPDH }\end{array}$ \\
& & $\begin{array}{l}\text { Forward: 5'-AGGTCGGTGTGAACGGATTTG-3' } \\
\text { Reverse: 5'-GGGGTCGTTGATGGCAACA-3' }\end{array}$ \\
\hline
\end{tabular}

examination was randomly divided into 4 groups (10 per group); normal control (NC), diabetes model (DM), low dose puerarin (LP) and high dose puerarin group (HP). All rats were housed in the SPF system and were fed a normal diet. Streptozotocin (STZ) (Sigma-Aldrich, St. Louis, MO, USA) induction was used to establish the diabetic rat model. Rats were fasted for $12 \mathrm{~h}$ before modeling, and STZ [10 g/l solution with $0.1 \mathrm{mmol} / \mathrm{LPBS}$ buffer $(\mathrm{pH} 4.5)]$ was injected intraperitoneally at a dosage of $60 \mathrm{mg} / \mathrm{kg}$. Seventy-two hours after injection, tail vein blood was collected for rapid blood glucose determination (if the tail vein blood glucose for rapid/l, the modeling was determined successful). After successful modeling, rats from each group were given intragastric administration once a day for 4 weeks; the NC and DM groups were given sterile normal saline $(10 \mathrm{ml} / \mathrm{kg})$, the LP group was given $250 \mathrm{mg} / \mathrm{kg}$ puerarin (Beijing Sihuan Pharmaceutical Co., Ltd., Beijing, China; Chinese medicine H20055195); and the HP group was given $500 \mathrm{mg} / \mathrm{kg}$ puerarin (13). At the end of the second and fourth week of intragastric administration, tail vein blood glucose was determined. The study was approved by the Ethics Committee of the Eye Hospital of Wenzhou Medical University.

The determination of electroretinogram (visual electrophysiological detector; Chongqing Kanghua Co., Ltd., Chongqing, China)] was performed before modeling and at 4 weeks before sacrifice. Rats were subjected to dark adaptation for $30 \mathrm{~min}$ and were anesthetized with intraperitoneally injected $3 \%$ pentobarbital sodium $(40 \mathrm{mg} / \mathrm{kg}$ ) followed by $0.5 \%$ of tropicamide/phenylephrine for ocular dilation. A ground electrode was connected to the tip of the ear skin, and the reference electrode was connected to the midpoint of the eyes subcutaneously. Then, white light shined for $200 \mathrm{~ms}$, at an intensity of $10 \mathrm{cds} / \mathrm{m}^{2}$, and the b-wave amplitude was recorded when the strongest reaction of the eyes was detected.

Prior to sacrifice, and after electroretinogram determination, abdominal aorta blood was drawn, and centrifuged to isolate serum that was stored at $-80^{\circ} \mathrm{C}$ for later determination of serum insulin, superoxide dismutase (SOD), malondialdehyde (MDA), and total anti-oxidation capacity (T-AOC) [using a kit (Nanjing Institute of Bioengineering, Nanjing, China)]. Both bulbus oculi were removed and placed in ice saline. Under the surgical microscope (Suzhou Liuliu vision Co., Ltd., Suzhou, China), both sides of retinal tissues were separated and stored at $-80^{\circ} \mathrm{C}$; one side was used for the determination of SOD, MDA and T-AOC, and the other side was used for the determination of STAT3 expression.

SOD, MDA, T-AOC and insulin in each group was determined from serum from each rat using appropriate kits and the manufacturer's protocol. The level of SOD was measured at $550 \mathrm{~nm}$, level of MDA was measured at $532 \mathrm{~nm}$, and the level of T-AOC was measured at $520 \mathrm{~nm}$ by continuous wavelength multi-functional microplate reader (Continuous wavelength multifunctional microplate reader; Tecan Austria $\mathrm{GmbH}$, Grödig, Austria). The determination of insulin (insulin ELISA kit; ALPCO, Salem, NH, USA) was determined per the kit protocol, and continuous wavelength multi-functional microplate reader was used to measure OD value; a standard curve was generated and insulin concentration was calculated.

Determination of the levels of SOD, MDA and T-AOC in retinal tissue homogenate of each group were determine. After the retinal tissue was weighed, normal ice saline was added at a ratio of 1:99, and the supernatant was collected after the homogenate was centrifuged. The ingredients were added according to the SOD, MDA and T-AOC assay kits and the specific determination method was the same as the one for serum.

Extraction of retinal RNA, and the reverse transcription (reverse transcription kit; Toyobo Co., Ltd., Tokyo, Japan) of retinal tissue was performed; an appropriate amount of TRIzol reagent (Takara Bio, Shiga, Japan) was added to extract total RNA. Ultraviolet spectrophotometer (ultrasonic ultraviolet spectrophotometer; Thermo Fisher Scientific, Waltham, MA, USA) was used to determine RNA concentration and purity. RNA underwent reverse transcription as follows: $2 \mu \mathrm{g}$ of RNA underwent heat denaturation for $5 \mathrm{~min}$ at $65^{\circ} \mathrm{C}$, and then immediately put on ice for cooling; then $4 \mu \mathrm{l}$ of $5 \mathrm{X}$ RT Master Mix was added, followed by DEPC treated water to reach a total volume of $20 \mu \mathrm{l}$ with reaction parameters were $37^{\circ} \mathrm{C} 15 \mathrm{~min}, 52^{\circ} \mathrm{C} 5 \mathrm{~min}, 98^{\circ} \mathrm{C} 5 \mathrm{~min}$. Real-time PCR was used to determination retinal STAT3 $\mathrm{mRNA}$ expression. The reaction system was as follows: $2 \mu \mathrm{l}$ cDNA, $0.5 \mu \mathrm{l}$ upstream and downstream primers (Table I for STAT3 and GAPDH primer sequences) and 12.5 $\mu 1$ XX SYBR-Green PCR Master Mix (SYBR-Green PCR Master Mix; Takara Bio) and ultrapure water was added to reach a total volume of $25 \mu \mathrm{l}$. Samples were analyzed a by real-time fluorescence quantitative PCR machine (real-time fluorescence quantitative PCR; Eppendorf, Hamburg, Germany); reaction parameters were as follows: $95^{\circ} \mathrm{C}$ for $30 \mathrm{sec}, 95^{\circ} \mathrm{C}$ for $5 \mathrm{sec}$, and $60^{\circ} \mathrm{C}$ for $30 \mathrm{sec}$ for 40 cycles, and the amplification curves and $\mathrm{Ct}$ values for each reaction were read [GAPDH (GAPDH primer; Shanghai Biomedical Engineering Co., Ltd., Shanghai, China) was used as the reference, the relative quantification $2^{-\Delta \Delta C t}$ method was used to compare the expression of each gene].

ELISA detection of retinal STAT3 protein was performed using a kit (STAT3 ELISA kit; R\&D Systems, Minneapolis, MN, USA), according to the manufacturer's protocol. To an appropriate amount of retina by weight was added normal 


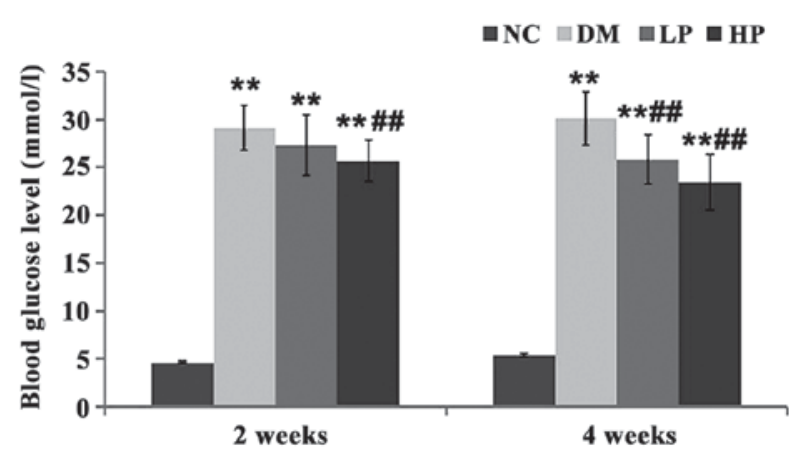

Figure 1. Comparison of blood glucose levels in each group. Compared with the NC group, blood glucose was significantly increased in DM, LP and HP groups at 2 and 4 weeks after modeling, but was significantly lower in HP group than in DM group. (Compared with $\mathrm{NC}$ group, ${ }^{* * *} \mathrm{P}<0.01$; compared with DM group, $\left.{ }^{\# \#} \mathrm{P}<0.01\right)$.

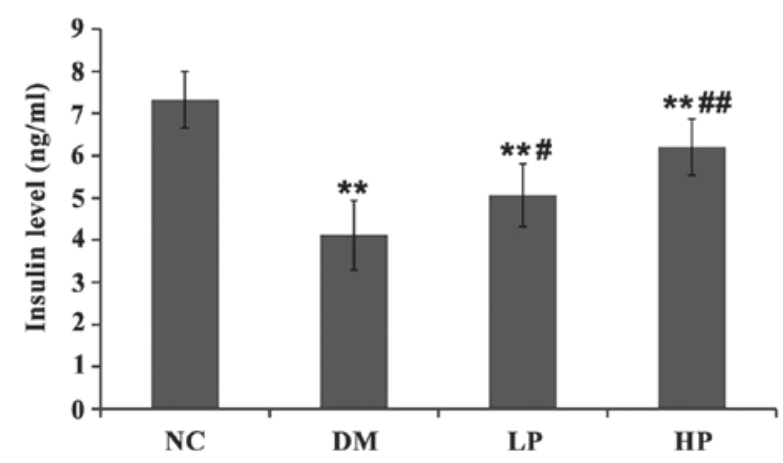

Figure 2. Comparison of insulin levels in each group. Compared with the NC group, DM, LP and HP groups showed significantly reduced insulin levels; in the LP and HP groups the insulin levels were significantly higher than that in the DM group. (Compared with the NC group, ${ }^{* *} \mathrm{P}<0.01$; compared with the $\mathrm{DM}$ group, $\left.{ }^{\#} \mathrm{P}<0.05,{ }^{\# \#} \mathrm{P}<0.01\right)$.

saline for homogenization; samples were centrifuged and the supernatant separated for ELISA. The OD value was measured by a continuous wavelength multifunctional microplate reader, a standard curve was drawn and the STAT3 protein content of each sample was calculated.

Results were statistically compared and shown as mean \pm SD, using the SPSS 20.0 statistical software (IBM, Armonk, NY, USA). An independent sample t-test was used for comparison between two groups, and one-way ANOVA was used to compare between multiple groups. $\mathrm{P}<0.05$ was used as the threshold to determine statistical significance.

\section{Results}

After STZ induction, the levels of blood glucose and insulin (Figs. 1 and 2) in the DM, LP and HP groups were significantly higher than those in the $\mathrm{NC}$ group $(\mathrm{P}<0.01)$. The blood glucose in the puerarin group was significantly lower than that in the DM group $(\mathrm{P}<0.01)$, and the insulin level was significantly higher, especially in the HP group $(\mathrm{P}<0.05)$.

There was no significant difference in $b$-wave amplitude of electroretinogram (Table II) between the groups before modeling $(\mathrm{P}>0.05)$. After treatment, the amplitude of $b$-wave of electroretinogram in the DM and LP groups were significantly lower than that in the NC group $(\mathrm{P}<0.01)$. Compared to
Table II. The comparison of b-wave amplitude of rat electroretinogram (mean $\pm \mathrm{SD}, \mu \mathrm{V})$.

\begin{tabular}{lll}
\hline Group & Before modeling & After treatment \\
\hline NC & $183.24 \pm 16.10$ & $178.73 \pm 19.21$ \\
DM & $176.19 \pm 19.21$ & $124.04 \pm 21.98^{\mathrm{a}}$ \\
LP & $175.66 \pm 21.53$ & $147.56 \pm 18.26^{\mathrm{a}, \mathrm{b}}$ \\
HP & $186.08 \pm 18.45$ & $163.30 \pm 22.15^{\mathrm{c}}$ \\
\hline
\end{tabular}

Compared with $\mathrm{NC}$ group, ${ }^{\mathrm{a}} \mathrm{P}<0.01$; compared with $\mathrm{DM}$ group, ${ }^{\mathrm{b}} \mathrm{P}<0.05,{ }^{\mathrm{c}} \mathrm{P}<0.01$.

Table III. Comparison of serum SOD, MDA and T-AOC levels in rats (mean $\pm \mathrm{SD})$.

\begin{tabular}{lrll}
\hline Group & SOD $(\mathrm{U} / \mathrm{ml})$ & MDA $(\mu \mathrm{mol} / \mathrm{l})$ & T-AOC $(\mathrm{U} / \mathrm{ml})$ \\
\hline NC & $124.07 \pm 12.13$ & $18.02 \pm 3.28$ & $14.54 \pm 1.86$ \\
DM & $83.22 \pm 10.25^{\mathrm{a}}$ & $27.10 \pm 2.09^{\mathrm{a}}$ & $12.48 \pm 1.67$ \\
LP & $98.91 \pm 9.38^{\mathrm{a}, \mathrm{c}}$ & $24.63 \pm 1.96^{\mathrm{a}, \mathrm{b}}$ & $13.79 \pm 2.00$ \\
HP & $115.60 \pm 11.25^{\mathrm{c}}$ & $20.79 \pm 2.55^{\mathrm{c}}$ & $12.93 \pm 1.58$ \\
\hline
\end{tabular}

Compared with $\mathrm{NC}$ group, ${ }^{\mathrm{a}} \mathrm{P}<0.01$; compared with $\mathrm{DM}$ group, ${ }^{\mathrm{b}} \mathrm{P}<0.05,{ }^{\mathrm{c}} \mathrm{P}<0.01$.

Table IV. Comparison of SOD, MDA and T-AOC levels in rat retinal tissues (mean $\pm \mathrm{SD})$.

\begin{tabular}{llll}
\hline Group & SOD $(\mathrm{U} / \mathrm{mg})$ & $\operatorname{MDA}(\mu \mathrm{mol} / \mathrm{g})$ & T-AOC $(\mathrm{U} / \mathrm{mg})$ \\
\hline $\mathrm{NC}$ & $159.20 \pm 13.27$ & $5.57 \pm 0.66$ & $2.46 \pm 0.41$ \\
$\mathrm{DM}$ & $117.36 \pm 14.13^{\mathrm{a}}$ & $9.20 \pm 1.00^{\mathrm{a}}$ & $1.82 \pm 0.33^{\mathrm{a}}$ \\
LP & $135.78 \pm 12.08^{\mathrm{a}, \mathrm{c}}$ & $7.61 \pm 0.87^{\mathrm{a}, \mathrm{c}}$ & $1.95 \pm 0.37$ \\
HP & $147.60 \pm 13.46^{\mathrm{c}}$ & $6.43 \pm 1.12^{\mathrm{c}}$ & $2.29 \pm 0.32^{\mathrm{b}}$ \\
\hline
\end{tabular}

Compared with $\mathrm{NC}$ group, ${ }^{\mathrm{a}} \mathrm{P}<0.01$; compared with $\mathrm{DM}$ group, ${ }^{\mathrm{b}} \mathrm{P}<0.05,{ }^{\mathrm{c}} \mathrm{P}<0.01$.

the DM group, the amplitude of b-wave of electroretinogram in the LP and HP groups was significantly higher $(\mathrm{P}<0.05)$, where the HP group showed a level close to normal $(\mathrm{P}>0.05)$.

The serum levels of SOD, MDA and T-AOC levels in rats were compared (Table III). The activity of SOD in the DM and LP groups was significantly lower than that in the NC group $(\mathrm{P}<0.01)$. The MDA content was significantly higher than that in the NC group $(\mathrm{P}<0.01)$. The SOD activity in the LP and HP groups was significantly higher than in the DM group $(\mathrm{P}<0.01)$, and the MDA level in those two groups was significantly lower than in the DM group $(\mathrm{P}<0.05)$. There was no significant difference between the HP group and the NC group $(\mathrm{P}>0.05)$. There was no significant difference in the serum T-AOC level among the groups $(\mathrm{P}>0.05)$.

The SOD, MDA and T-AOC levels in rat retinal tissues were compared (Table IV). The activity of SOD in the DM and 


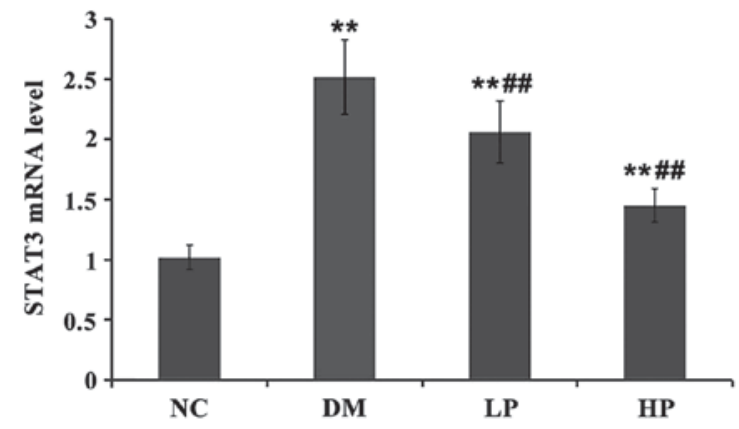

Figure 3. Compared with NC group, STAT3 mRNA levels in DM, LP and HP groups were significantly higher. Compared with the DM group, STAT3 mRNA levels in LP and HP groups were significantly lower. (Compared with NC group, ${ }^{* *} \mathrm{P}<0.01$; compared with $\mathrm{DM}$ group, $\left.{ }^{\# \#} \mathrm{P}<0.01\right)$.

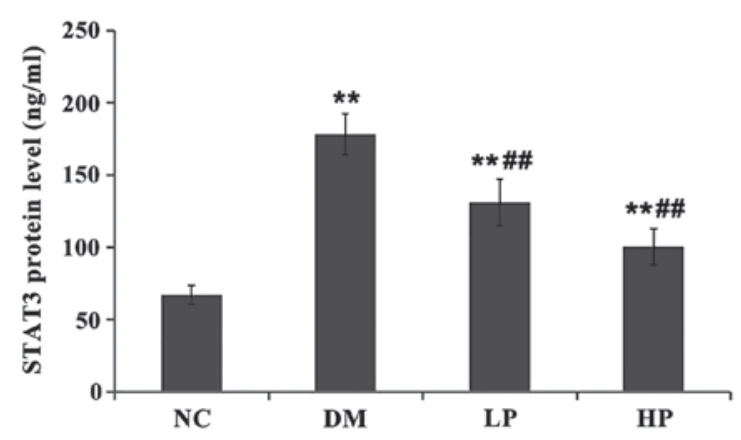

Figure 4. Compared with the control group, the STAT3 protein levels in DM, LP and HP groups were significantly higher. Compared with DM group, the STAT3 protein levels in LP and HP groups were significantly lower. (Compared with NC group, ${ }^{* *} \mathrm{P}<0.01$; compared with DM group, ${ }^{\#} \mathrm{P}<0.01$ ).

LP groups was significantly lower than that in the NC group $(\mathrm{P}<0.01)$, but the MDA content was significantly higher than that in the NC group $(\mathrm{P}<0.01)$; the activity of SOD in the LP and HP groups was significantly higher than in the DM group $(\mathrm{P}<0.01)$ but the MDA level was significantly lower than in the DM group. There was no significant difference between the HP group and the NC group $(\mathrm{P}>0.05)$. The $\mathrm{T}-\mathrm{AOC}$ in retina tissues in the DM group was significantly lower than that in the NC group $(\mathrm{P}<0.01)$. Retinal T-AOC was significantly higher in the HP group than in the DM group $(\mathrm{P}<0.05)$, while the effect of low-dose puerarin did not achieve statistical significance.

The level of STAT3 mRNA in rat retinal tissue between groups was compared (Fig. 3). Compared with the NC group, STAT3 mRNA levels in the DM, LP and HP groups were significantly higher $(\mathrm{P}<0.01)$. Compared with the DM group, STAT3 mRNA levels in the LP and HP groups were significantly lower $(\mathrm{P}<0.01)$. The protein level of STAT3 in rat retinal tissue was compared (Fig. 4). Compared with the control group, the STAT3 protein levels in the DM, LP and HP groups were significantly higher $(\mathrm{P}<0.01)$.

\section{Discussion}

As the incidence of type 2 diabetes continues to increase, so does the complication of DR. Due to an abnormal sustained high serum glucose state, a proliferation in both vascular smooth muscle cells and endothelial cells occurs, along with an increase in vascular permeability. The high glucose state also activates the body's stress and inflammatory responses that can further cause ganglion cell damage and apoptosis $(14,15)$. DR, as a complication from diabetes, is a small vessel disease. The current understanding of the pathogenesis of DR is due to a large number of glycosylated products deposited in the retinal capillaries, basement membrane and peripheral cells, that cause retinal inflammation and oxidative stress response, resulting in platelets chemotaxis. Leukocytes and platelet aggregation occurs in the retinal localized vessels, eventually leading to retinal vascular occlusion (16). Subsequent inflammation and oxidative stress are key to the occurrence and development of DR (17). The sustained high serum glucose state can inhibit the antioxidant system in vivo, causing the antioxidant enzymes to glycosylate, antioxidant enzyme (e.g. catalase, SOD) activity to decrease resulting in a weakened system for of free radical scavenging (18). This study also confirmed that the serum and retinal tissue of DM rats showed lower SOD and T-AOC levels, lipid peroxidation product (MDA) accumulation and severe oxidative stress in the body.

Its mechanism puerarin in the modulation of diabetes and DR includes a hypoglycemic effect, improvement of insulin resistance, decreased oxidative stress, promotion of retinal microcirculation, improvement of blood rheology, inhibition of platelet aggregation, and reduction in plasma viscosity, thereby inhibiting thrombosis (19). Concurrently, puerarin can improve the microcirculation in retinal tissue, reduce the expression of inflammatory mediators during retinal damage, and, therefore, protect the retina ultrastructure $(8,20)$. Chen et al $(21)$ found that puerarin can increase the activity of SOD in serum and retinal tissue of DM rats, increase the levels of glutathione peroxidase, catalase and nitric oxide, decrease the level of MDA in DM rats, and improve the animal's antioxidant capacity. Results from this investigation, also observed that puerarin can reduce blood glucose in DM rats, increase insulin levels, increase serum and retinal SOD activity in DM rats, improve retinal T-AOC content, and reduce MDA in serum and retinal tissue (more so with the higher dose used). These results suggest that puerarin can enhance the antioxidant capacity of the retina, and thus play a protective role for visual function.

The electroretinogram b-wave originates from the outer layer of the retina, and its changes can reflect the functional status of the retinal neurons downstream from the retinal ganglion (22). An animal study found that (22), using the STZ-induced DM rat model, b-wave amplitude started decreasing 2 weeks after modeling. Results from this investigation also observed that 4 weeks after the modeling, DM rats showed a significant decrease in b-wave amplitude, indicating early retinal nerve cell damage. After puerarin intervention, the amplitude of retinal b-wave was significantly higher than that of DM group, suggesting that puerarin had a protective effect on the retinal neurons.

STAT3 is a DNA-binding cytoplasmic transcription factor that is involved in nuclear translocation phosphorylation, and is widely expressed in the retina and other tissues (23). STAT3 is activated by dimerization or targeted phosphorylation, and then participates in signaling pathways such as the JAK/STAT signaling pathway (23) which is involved in a series of biological functional roles (6). STAT3 is also involved in the occurrence 
and development of DR. Joussen et al (24) found that in early DM rat retina, STAT3 increased after the first day, reached the peak after 1 week and decreased gradually in following weeks. One explanation that DR causes visual impairment is that due to the increase in retinal capillary permeability, which leads to inflammation and retinal edema, ultimately causing visual impairment. In this process, vascular endothelial growth factor plays a key role (25). That study found that under high glucose toxicity, activity of reactive oxygen species are increased, causing phosphorylation of retinal capillary endothelial cells in JAK2/STAT3, thereby increasing the expression of vascular endothelial growth factor. By inhibiting the phosphorylation of JAK2/STAT3, the expression of vascular endothelial growth factor can be reduced, thereby preventing the occurrence of DR (26). Results from this investigation found that STAT3 in DM rats was significantly higher than in normal rats, and puerarin can effectively inhibit the expression of STAT3, and play a role in reducing DR. The mechanism of the effect is hypothesized to be due to inhibition of the phosphorylation of JAK2/STAT3, thereby reducing the expression of vascular endothelial growth factor and the inflammation of the retina, and that results in preventing the occurrence of DR.

In summary, puerarin can decrease the blood glucose of DM rats, increase the level of insulin, increase the activity of SOD in serum and retina of DM rats, increase the content of T-AOC in the retina, decrease the content of MDA in the serum and retina, and therefore enhance the antioxidant ability of the retina. Concurrently, puerarin can significantly inhibit the expression of STAT3, and play a role in reducing DR. The occurrence of DR involves a variety of factors and complex regulatory mechanisms, which include the role of inflammatory cytokines, and the details of the specific cell signaling pathways require further investigation. Controlled clinical trials are needed to determine if these effects from puerarin in the animal model translate to comparable results in diabetic patients.

\section{Acknowledgements}

The present study was supported by the Wenzhou Science and Technology Planning Project in the Public Interest (Y20150372).

\section{References}

1. Frank RN: Diabetic retinopathy. N Engl J Med 350: 48-58, 2004

2. Wang FH, Liang YB, Zhang F, Wang JJ, Wei WB, Tao QS, Sun LP, Friedman DS, Wang NL and Wong TY: Prevalence of diabetic retinopathy in rural China: The Handan Eye Study. Ophthalmology 116: 461-467, 2009.

3. Kempen JH, O'Colmain BJ, Leske MC, Haffner SM, Klein R, Moss SE, Taylor HR and Hamman RF; Eye Diseases Prevalence Research Group: The prevalence of diabetic retinopathy among adults in the United States. Arch Ophthalmol 122: 552-563, 2004.

4. Roy MS, Klein R, O'Colmain BJ, Klein BEK, Moss SE and Kempen JH: The prevalence of diabetic retinopathy among adult type 1 diabetic persons in the United States. Arch Ophthalmol 122: 546-551, 2004.

5. Arden GB and Sivaprasad S: The pathogenesis of early retinal changes of diabetic retinopathy. Doc Ophthalmol 124: 15-26, 2012 .

6. Zhong Z, Wen Z and Darnell JE Jr: Stat3: A STAT family member activated by tyrosine phosphorylation in response to epidermal growth factor and interleukin-6. Science 264: 95-98, 1994.
7. Chistiakov DA: Diabetic retinopathy: Pathogenic mechanisms and current treatments. Diabetes Metab Syndr 5: 165-172, 2011.

8. Chang Y, Hsieh CY, Peng ZA, Yen TL, Hsiao G, Chou DS, Chen CM and Sheu JR: Neuroprotective mechanisms of puerarin in middle cerebral artery occlusion-induced brain infarction in rats. J Biomed Sci 16: 9, 2009.

9. Wang N, Zhang Y, Wu L, Wang Y, Cao Y, He L, Li X and Zhao J: Puerarin protected the brain from cerebral ischemia injury via astrocyte apoptosis inhibition. Neuropharmacology 79: 282-289, 2014.

10. Wang X, Li S, Li J, Li C and Zhang Y: De novo transcriptome sequencing in Pueraria lobata to identify putative genes involved in isoflavones biosynthesis. Plant Cell Rep 34: 733-743, 2015.

11. She S, Liu W,Li T and Hong Y: Effects of puerarin in STZ-induced diabetic rats by oxidative stress and the TGF- $\beta 1 / \mathrm{Smad} 2$ pathway. Food Funct 5: 944-950, 2014

12. Zhong Y,Zhang X, Cai X, Wang K, Chen Y and Deng Y: Puerarin attenuated early diabetic kidney injury through down-regulation of matrix metalloproteinase 9 in streptozotocin-induced diabetic rats. PLoS One 9: e85690, 2014.

13. Mao CP and Gu ZL: Experimental effect of puerarin on the formation of advanced glycation end products and expression of RAGE in the arota or diabetic rats. Chin Pharm Bulletin 20: 393-397, 2005.

14. Hampton BM, Schwartz SG, Brantley MA Jr and Flynn HW Jr: Update on genetics and diabetic retinopathy. Clin Ophthalmol 9: 2175-2193, 2015.

15. Kovacs K, Marra KV, Yu G, Wagley S, Ma J, Teague GC, Nandakumar N, Lashkari K and Arroyo JG: Angiogenic and inflammatory vitreous biomarkers associated with increasing levels of retinal ischemia. Invest Ophthalmol Vis Sci 56: 6523-6530, 2015

16. Chen Y: The pathologic mechanism of diabetic retinopathy. Int J Ophthalmol 6: 433-435, 2006.

17. Du XL, Edelstein D, Rossetti L, Fantus IG, Goldberg H, Ziyadeh F, Wu J and Brownlee M: Hyperglycemia-induced mitochondrial superoxide overproduction activates the hexosamine pathway and induces plasminogen activator inhibitor-1 expression by increasing Spl glycosylation. Proc Natl Acad Sci USA 97: 12222-12226, 2000.

18. Maritim AC, Sanders RA and Watkins JB III: Diabetes, oxidative stress, and antioxidants: A review. J Biochem Mol Toxicol 17: 24-38, 2003.

19. Wu K, Liang T, Duan X, Xu L, Zhang K and Li R: Anti-diabetic effects of puerarin, isolated from Pueraria lobata (Willd.), on streptozotocin-diabetogenic mice through promoting insulin expression and ameliorating metabolic function. Food Chem Toxicol 60: 341-347, 2013.

20. Li JC, Jiang YX, Zhang SY, Wang L, Ouyang YS and Qi ZH: Evaluation of renal artery stenosis with hemodynamic parameters of Doppler sonography. J Vasc Surg 48: 323-328, 2008.

21. Chen F, Zhang HQ, Zhu J, Liu KY, Cheng H, Li GL, Xu S, Lv WH and Xie ZG: Puerarin enhances superoxide dismutase activity and inhibits RAGE and VEGF expression in retinas of STZ-induced early diabetic rats. Asian Pac J Trop Med 5: 891-896, 2012.

22. Li Q, Zemel E, Miller B and Perlman I: Early retinal damage in experimental diabetes: Electroretinographical and morphological observations. Exp Eye Res 74: 615-625, 2002.

23. Calò V, Migliavacca M, Bazan V, Macaluso M, Buscemi M, Gebbia N and Russo A: STAT proteins: From normal control of cellular events to tumorigenesis. J Cell Physiol 197: 157-168, 2003.

24. Joussen AM, Huang S, Poulaki V, Camphausen K, Beecken WD, Kirchhof B and Adamis AP: In vivo retinal gene expression in early diabetes. Invest Ophthalmol Vis Sci 42: 3047-3057, 2001.

25. Caldwell RB, Bartoli M, Behzadian MA, El-Remessy AE, Al-Shabrawey M, Platt DH, Liou GI and Caldwell RW: Vascular endothelial growth factor and diabetic retinopathy: Role of oxidative stress. Curr Drug Targets 6: 511-524, 2005.

26. Ushio-Fukai M, Tang Y, Fukai T, Dikalov SI, Ma Y, Fujimoto M, Quinn MT, Pagano PJ, Johnson C and Alexander RW: Novel role of gp91(phox)-containing NAD(P)H oxidase in vascular endothelial growth factor-induced signaling and angiogenesis. Circ Res 91: 1160-1167, 2002. 